\title{
Consolidation of crossmodal correspondences between auditory pitch and spatial features in childhood
}

\author{
Irune Fernández-Prieto ${ }^{1,2^{*}}$, Ferran Pons ${ }^{2,3}$ and Jordi Navarra ${ }^{3}$
}

$1 \mathrm{CIMeC}$, Center for Mind/Brain Sciences, University of Trento, Italy

2 Institute of Neurosciences, University of Barcelona, Barcelona, Spain

3 Department of Cognition, Development and Educational Psychology,

University of Barcelona, Barcelona, Spain

*Corresponding author: Irune Fernández-Prieto, Center for Mind/Brain Sciences,

University of Trento, Corso Bettini, 31, 38068 Rovereto (TN), Italy

E-MAIL: irunefernandez@gmail.es 


\begin{abstract}
Crossmodal correspondences between auditory pitch and spatial elevation have been demonstrated extensively in adults. High- and low-pitched sounds tend to be mapped onto upper and lower spatial positions, respectively. We hypothesised that this crossmodal link could be influenced by the development of spatial and linguistic abilities during childhood. To explore this possibility, 70 children (9-12 years old) divided into three groups (4th, 5th and 6th grade of primary school) completed a crossmodal test to evaluate the perceptual correspondence between pure tones and spatial elevation. Additionally, we addressed possible correlations between the students' performance in this crossmodal task and other auditory, spatial and linguistic measures. The participants' auditory pitch performance was measured in a frequency classification test. The participants also completed three tests of the Wechsler Intelligence Scale for Children-IV (WISC-IV): (1) Vocabulary, to assess verbal intelligence, (2) Matrix reasoning, to measure visuospatial reasoning and (3) Blocks design, to analyse visuospatial/motor skills. The results revealed crossmodal effects between pitch and spatial elevation. Additionally, we found a correlation between the performance in the block design subtest with the pitch-elevation crossmodal correspondence and the auditory frequency classification test. No correlation was observed between auditory tasks with matrix and vocabulary subtests. This suggests (1) that the crossmodal correspondence between pitch and spatial elevation is already consolidated at the age of 9 and also (2) that good performance in a pitch-based auditory task is mildly associated, in childhood, with good performance in visuospatial/motor tasks.
\end{abstract}


Audiospatial crossmodal associations in children

Keywords: crossmodal correspondences; pitch perception; visuospatial abilities, language

\section{Research highlights}

- We explore the crossmodal link between pitch and spatial elevation in children.

- We seek to investigate whether spatial and linguistic abilities influence this crossmodal link.

- This pitch-spatial elevation link is already consolidated at the age of 9 .

- Verbal skills are not an essential factor for the emerge of this association link.

- Performance in a pitch-based task is related to performance in spatial tasks. 


\section{Introduction}

In our everyday life, the brain creates coherent representations of information from multiple sensory modalities. These crossmodal associations (so-called crossmodal correspondences) involve the interaction between sensory features from different modalities (e.g., visual size and auditory pitch). There is a consistent perceptual association between different visuospatial features (e.g., visuospatial elevation or size) and sound (e.g., pitch or loudness). For example, we tend to associate high and low frequency sounds with objects located in high and low positions, respectively (Deroy, Fernández-Prieto, Navarra, \& Spence, 2018; Spence, 2011). In a seminal study, Melara and O'Brien (1987) found crossmodal correspondences between visuospatial elevation (e.g., upper and lower positions) and pitch (e.g., high and low pitch). The participants in this study responded faster when both dimensions were congruent (e.g., upper position of the visual object and higher-pitched sounds). Rusconi, Kwan, Giordano, and Umilta (2006) explored the extent at which sound frequencies could be spatially mapped in vertical coordinates. These authors designed a speeded classification task where the participants had to compare the frequency of two different tones by pressing one of two keys located on a "symbolic" vertical axis (for example, "up" or "down"). The results of this study revealed that participants were faster and more accurate responding to the high and low tones when the answer corresponded to the location of the key located "up" and "down" on the keyboard, respectively (see also Occelli, Spence, \& Zampini, 2009). Douglas and Bilkey (2007) indirectly explored whether performing pitch-based and specific visuospatial tasks (e.g., mental rotation) involved, at least at certain processing stages, similar cognitive mechanisms as well as brain structures. These authors found that patients with amusia (also known as tonedeafness) had worse performances on mental rotation tasks, compared to a healthy control group (however, see Tillmann et al., 2010 for contradicting results). Thus, it may be the case that brain areas involved in spatial processing are also involved in the 
analysis of sound pitch, especially during the completion of musical tasks such as the detection of pitch violations in transposed melodies (Foster \& Zatorre, 2010a; Foster \& Zatorre, 2010b).

Although less consistently than in adults, audio-visual associations have also been observed in infants and children (Mondloch and Maurer, 2004; Walker et al., 2010; Dolscheid et al., 2014; Fernández-Prieto, Navarra and Pons, 2015; Nava, Grassi and Turati, 2016). For example, Walker et al. (2012) found that 3- to 4-month-old infants were sensitive to specific audio-visual associations. In a preferential-looking task, infants watched an animation showing a ball moving up and down presented with a sound with ascending $(300$ to $1700 \mathrm{~Hz})$ or descending frequency $(1700$ to $300 \mathrm{~Hz})$. Infants looked longer to the screen in congruent trials, in which visuospatial height and sound matched (e.g., the ball moving up together with a sound with ascending frequency) than to the incongruent ones. However, the idea of crossmodal correspondences as an innate aspect of perception is still controversial. Despite this research evidence suggesting the presence of crossmodal effects in the earlier stages of life, other studies have failed to replicate these crossmodal effects. Lewkowicz and Minar (2014) did not observe a link between auditory pitch and visuospatial features in 4- to 14-month-old infants. It is possible that crossmodal associations in infants could be less consistent due to the premature development of neural mechanisms that support the integration of different sensory systems during the first years of life (Desjardins, Rogers, \& Werker, 1997; Desjardins \& Werker, 2004; Massaro, 1984; Massaro, Thompson, Barron, \& Laren, 1986). Moreover, the mechanisms of the various sensory systems develop at different velocities: tactile, auditory, or chemical processing (i.e., taste and smell) begin to function in the maternal womb, while visual processing is a modality that does not reach full functionality until 12 months after birth (for a review, see Burr \& Gori, 2012). This disparity in sensory development could delay the efficient integration of multisensory information in the early stages of life. 
Multisensory audio-visual integration continues its development throughout childhood, especially during primary school. Interestingly, the ability to reduce uncertainty and improve perceptual precision by integrating information from different sensory modalities evolves from infancy through childhood and into adulthood. Integration of multisensory perceptual information appears to develop from 7 to 12 years (e.g., visualhaptic size discrimination) (Gori et al., 2008; Barutchu, Crewther and Crewther, 2009; Nardini, Bales and Mareschal, 2016).

Another relevant aspect regarding audio-visual crossmodal associations is the role of language on its appearance. While studies conducted with adults clearly show the presence of a crossmodal association between pitch (i.e., high vs low pitch) and vertical elevation (up vs down spatial positions), studies with prelinguistic infants present somewhat contradicting results (Dolscheid et al., 2014; Lewkowicz \& Minar, 2014; Walker et al., 2010). Parkinson, Kohler, Sievers, and Wheatley (2012) addressed the role of language in crossmodal correspondences. To do so, they tested a group of adult participants from the Kreung tribe (Cambodia), whose language does not include spatial-related labels (e.g., "high" or "low") to classify pitch. The participants showed crossmodal correspondences between verticality and pitch, indicating that language may not be indispensable for these perceptual association to occur. This evidence may perhaps compete with Martino and Marks' semantic coding hypothesis (1999), where the crossmodal correspondences are mediated, at some level, by higher-order, and language-mediated, mechanisms, and also with Long (1977) in which it was suggested that crossmodal associations could emerge from the regularity of specific word use, for example, adopting the same linguistic label to describe an attribute in two different sensory modalities or sensory attributes (e.g., the word "high" to define the sound frequency and spatial location) (see also Gallace \& Spence, 2006). In a more recent study, Fernandez-Prieto, Spence, Pons and Navarra (2016) compared the possible influence of linguistic background on crossmodal associations in Spanish and English 
speakers. In English, "high" and "low" clearly denote both pitch and spatial elevation. By contrast, different words are preferentially used, in Spanish, for pitch (high: "agudo", low: "grave") and spatial verticality (high: "alto"; low: "bajo"). Both linguistic groups evidenced crossmodal correspondences. However, English speakers showed larger crossmodal effects between these two perceptual features, perhaps suggesting an influence of linguistic background on this crossmodal correspondence. Thus, it is plausible that linguistic experience could widely influence crossmodal associations. In the present study, we further explored the extent at which spatial elevation and pitch are perceptually associated in childhood and if linguistic factors modulate these associations.

Based on previous evidence suggesting a relationship between crossmodal correspondences and the maturity of the multisensory perceptual system (Nardini, Bales and Mareschal, 2016), we first predicted a gradual strength of crossmodal audiovisual associations related to age, in children. Moreover, we also expected a positive correlation between linguistic abilities and audio-visual crossmodal associations.

Taking into consideration that the development of pitch representation begins to appear around the age of six (Munar, Rosselló, Mas, Morente, \& Quetgles, 2002), and between 8 to 12 years of age the ability to integrate information from different sensory modalities is refined (Gori et al., 2008), we selected a group of children of 9- to 12years-old to test our hypotheses. The children carried out a Frequency classification test and a Pitch-elevation crossmodal correspondence test (adapted from Rusconi et al.'s study, 2006) to assess the possible link between space elevation and sound. To explore the possible associations between the performance in the auditory frequency classification with the audio-spatial classification, and the processing of spatial information (e.g. height) and linguistic abilities, participants also performed three different neuropsychological subtests from the Wechsler Intelligence Scale for Children-IV (WISC-IV): Block design, Matrix Reasoning, and Vocabulary. The "Block 
Audiospatial crossmodal associations in children

design subtest" measures spatial visualisation and visuomotor skills; the "Matrix reasoning subtest" assesses fluid visuospatial processing and reasoning; and the "Vocabulary subtest" measures verbal IQ (e.g., language development or verbal fluency).

\section{Materials and methods}

\section{Participants}

Seventy participants (thirty-two females, mean age $10.4 \pm S D 0.9$ years) from Can Cantó Public School in Ibiza (Spain) participated in this study. The participants belonged to three different group grades: (1) 28 students from 4th grade (twelve females, mean age $9.5 \pm$ SD 0.48 years), (2) 22 students from 5th grade (thirteen females, mean age $10.5 \pm S D 0.48$ years) and, (3) 20 students from $6^{\text {th }}$ grade (seven females, mean age $11.5 \pm$ SD 0.49 years).

All of the participants presented normal hearing and normal or corrected-to-normal vision. The data from children who either received speech or psychological treatment $(n=2)$ or were assessed as intellectual gifted $(n=2)$ were not included in the study. Finally, teachers confirmed that all students included in the study had an average academic performance and satisfactory linguistic development for their age.

\section{Apparatus}

An Intel Core laptop computer with a 15-inch monitor $(60 \mathrm{~Hz})$ was used for each of the computerised tests included in the study; mainly the Visuospatial classification test, the Auditory-spatial classification test and the three WISC-IV subtests. The testing was conducted individually in a quiet room in the school. The participants sat with their head 
approximately $60 \mathrm{~cm}$ from the monitor. The auditory stimuli were presented through headphones (Phillips SHP1900, China), at a comfortable listening level. The experimental procedure in all of the computerised tests was controlled by E-Prime 2.0 (Psychology Software Tools Inc., Pittsburg, PA). The participants' responses were obtained using a modified computer keyboard with a vertical orientation. The keyboard consisted of a flat panel with two response foam buttons located above and below a rest platform. The distance between the two response buttons was $15.7 \mathrm{~cm}$, and the rest platform was located at the same distance with respect to each of the two buttons, on the left side of the response board. The Pitch-elevation crossmodal correspondence test was conducted for each grade group in the classroom, using two speakers to present the auditory stimuli.

\section{Procedure}

First, all participants completed the Frequency classification test for $20 \mathrm{~min}$ approximately (see below) in a group session administered in each classroom separately due to school lesson time restrictions. The participants performed all of the other tasks individually for 45 minutes approximately, in a second session conducted during the subsequent days. The second session was divided into three blocks with a pause in between to reduce possible effects of fatigue and boredom. In the first and last blocks, the participants completed either the Pitch-elevation crossmodal correspondence test or the Visuospatial classification test with the order counterbalanced across participants. In the second block, the participants completed the three subtests of the WISC in random order. 
In an adaptation of Rusconi et al.'s (2006) study, participants had to compare, as quickly and accurately as possible, if a sound was higher or lower (low frequencies: 164.81 and $185.00 \mathrm{~Hz}$, and high frequencies: 369.99 and $415.30 \mathrm{~Hz}$ ) than a previously fixed reference sound $(261.63 \mathrm{~Hz})$. All sounds lasted for $1000 \mathrm{~ms}$. To respond, participants pressed one of two keys located in a superior or inferior part of the modified vertical keyboard (see Figure 1). The experiment was divided into two blocks. In the congruent block, participants had to press the upper and lower key when the second sound was higher or lower than the reference tone, respectively. In the incongruent block, participants had to press the upper and lower key when the second sound was lower or higher than the reference tone, respectively. The two blocks were presented in a counterbalanced order across all participants.

The participants completed 96 trials ( 24 trials for each comparison tone). Each trial began with a white fixation cross $(0.5 \times 0.5 \mathrm{~cm})$ that appeared in the centre of the computer screen for $250 \mathrm{~ms}$, on a black background. Subsequently, the reference sound was presented and, after $1500 \mathrm{~ms}$, one of the four possible sounds was played. Participants had to indicate with their dominant hand whether the sound was higher or lower than the reference one. Feedback (a text message: positive, negative or warning signal in case of no response) was provided $750 \mathrm{~ms}$ after the participant's response or after $3500 \mathrm{~ms}$ if no response was given.

The participants completed a training session before starting the main task. This session consisted of a simplified version of the task in which only two of the comparison tones (i.e., the ones with the lower and the higher tone; 165 and $415 \mathrm{~Hz}$ ) were used in 8 different trials per condition (incongruent and congruent). All participants completed the training session with no difficulties. 
a)

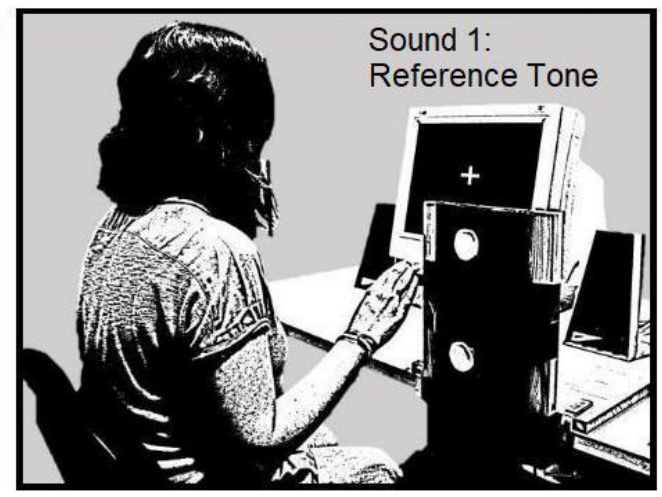

b)

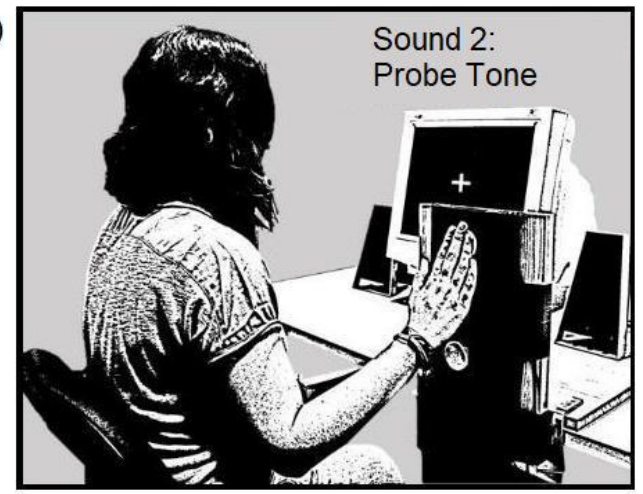

Figure 1. Experimental setup. (a) Participants had to place their hand on the starting position, a platform located between the two response buttons. (b) Participants responded whether a probe pitch was higher or lower than the reference tone by pressing one of two different buttons located above and below the starting position. Image under CC BY 4.0 license adapted from: Fernandez-Prieto I, Spence C, Pons F, Navarra J. Does Language Influence the Vertical Representation of Auditory Pitch and Loudness? i-Perception. June 2017. doi:10.1177/2041669517716183

\section{Visuospatial classification test}

This test was used to ensure that the participants perceived simple unisensory visuospatial information correctly. Therefore, high performance levels were expected in this test. A unimodal visual task (i.e., a visuospatial classification test) was designed with a procedure that mimicked the one used in the Pitch-elevation crossmodal correspondence test. Participants had to discriminate the spatial position of a grey circle (diameter: $2.5 \mathrm{~cm}$ ) (down positions: $0.75,1.5,2.25$ and $3 \mathrm{~cm}$ from the centre position, and high positions: $0.75,1.5,2.25$ and $3 \mathrm{~cm}$ from the centre position) from a central fixed reference yellow circle (diameter: $2.5 \mathrm{~cm}$ ). The procedure and the stimuli duration were identical to the Pitch-elevation crossmodal correspondence test, except for the addition of a completely grey screen that was presented for $700 \mathrm{~ms}$, between 
Audiospatial crossmodal associations in children

the central reference circle and the proof circle, to avoid the sense of movement between the reference circle and the posterior probe circle.

The participants completed a training session before starting the task. The training session consisted of a simplified version of the task in which only two of the comparison positions (i.e., the ones with the higher distance with respect to the centre, $3 \mathrm{~cm}$ upper and lower than the centre position) were used in 8 different trials per condition (incongruent and congruent). All participants completed the training session with no difficulties.

\section{Frequency classification test}

Participants listened to 14 pairs of sounds. A first and second sound were played consecutively with a duration of $1000 \mathrm{~ms}$ for each one. Each pair of sounds was played twice. The frequency distance between the sounds could be one musical tone or one semitone (within the range of $92 \mathrm{~Hz}$ to $698 \mathrm{~Hz}$ ). Participants had to answer in a paper questionnaire whether the second sound was higher or lower than the first sound for each trial. Instructions were given orally and were also available in the questionnaire. Before starting the main experiment, the participants completed a brief training block, consisting of a simplified version of the experimental task that included four examples of each possible pair of sounds with a clear difference in frequency (an octave or a fifth) between them (e.g., $440 \mathrm{~Hz}$ to $880 \mathrm{~Hz}$ ). A total score was obtained from this test, ranging from 0 ( $0 \%$ of correct answers) to 14 (100\% of correct answers).

\section{Block design subtest (from WISC-IV)}

Participants had to reproduce an image design displayed on the computer screen by using 4 or 9 bi-coloured plastic blocks (red and white). This standardised test had a 
Audiospatial crossmodal associations in children

time limit to reconstruct the image. The level of difficulty (i.e., the time given to reconstruct the image; $30,45,75$ or 120 seconds) increased each trial. The participants' score in this test ranged from 0 to 19 points.

Matrix reasoning subtest (from WISC-IV)

The participant's task was to complete a matrix formed by a partially filled grid.

Participants had to determine the correct sequence of a visual pattern (e.g., by colour, number, shape) with a missing figure. The correct figure had to be selected from five different options. The task was untimed. The participants' score in this test also ranged from 0 to 19 points.

Vocabulary subtest (from WISC-IV)

The participants were asked to give oral definitions of a series of words of increasing difficulty (e.g., umbrella, brave, amendment). This task was untimed, and the score also ranged from 0 to 19 points.

\section{Results}

In the Pitch-elevation crossmodal correspondence test and the Visual-spatial classification test, both the reaction times (RTs) and the number of errors were used to compare the performance of the participants. Reaction times faster than $150 \mathrm{~ms}$ were considered anticipatory responses and were removed from the analyses.

For the Pitch-elevation crossmodal correspondence test, a mixed, repeated-measures analysis of variance (ANOVA) test was used, including the between-subjects factor "Group" (4th, 5th and 6th grade) and the within-subjects factor "Congruency" 
(Congruent vs. Incongruent). The test revealed a significant main effect of Congruency for RTs $\left[F(1,67)=6.30, p=.01, n p^{2}=.09\right]$, and for number of errors $[F(1,67)=12.36$, $\left.p<.01, n p^{2}=.16\right]$, but no interaction between the two factors (Group and Congruency) in the RT's or the number of errors $[F(1,67)=.46, p=.96]$ and $[F(1,67)=.512, p=$ $.60]$, respectively.

Subsequently, to evaluate the relation between the performance in the Pitch-elevation crossmodal correspondence test and the frequency classification task with the linguistic and spatial subtests, Spearman rho coefficient was employed due to an absence of normality of the participants' data obtained in the Frequency classification test $(\mathrm{W}=$ $0.92, p<.01)$, Matrix reasoning subtest $(\mathrm{W}=0.96, p=.02)$, and the difference of Visuospatial classification test (Congruent minus Incongruent) $(\mathrm{W}=0.94, p<.01)$. The results revealed significant correlations between the Block design subtest and the Frequency classification test $(\rho=.35, p<.01 ; 95 \% \mathrm{Cl}=0.12-0.55)$, and the Block design subtest and the Pitch-elevation crossmodal correspondence test $(\rho=.24, p>$ $0.05 ; 95 \% \mathrm{Cl}=0.00-0.45)$. No correlation was found between the Pitch-elevation crossmodal correspondence test and Vocabulary Subtest $(\rho=.15, p=.21 ; 95 \% \mathrm{Cl}=-$ $0.09-0.37)$, and the Frequency classification test and Vocabulary subtest $(\rho=.18, p=$ $.13 ; 95 \% \mathrm{Cl}=-0.06-0.40)($ see Table 1 and Figure 2).

For the Visual-spatial classification test, an equivalent ANOVA was used, including the between-subjects factor "Group" $\left(4^{\text {th }}, 5^{\text {th }}\right.$ and $6^{\text {th }}$ grade $)$ and the within-subjects factor "Congruency" (Congruent vs. Incongruent). The analyses revealed a significant main effect of Congruency for RTs $\left[F(1,67)=79.05, p<.01, n p^{2}=.54\right]$, and for number of errors $\left[F(1,67)=48.05, \mathrm{p}<.01, n p^{2}=.43\right]$, but no interaction between the two factors in $\operatorname{RTs}\left[F(1,67)=0.14, p=.87, n p^{2}<.01\right]$, or number of errors $[F(1,67)=0.22, p=$ $\left..81, \eta p^{2}<.01\right]$. 
Audiospatial crossmodal associations in children

As expected, the performance in the Visual-spatial classification test was high. In the congruent condition, the average of correct responses was $96.46 \%(S D=4.04 \%)$ and in the incongruent condition, the average of correct responses was $90.67 \%$ ( $S D=$ $7.68 \%)$. 
Audiospatial crossmodal associations in children

Table 1. Spearman's rho correlations (2-tailed) from auditory, audio-visual and vocabulary tasks and all the tasks and subtests $(N=70)$. Parentheses represent $p$ values.

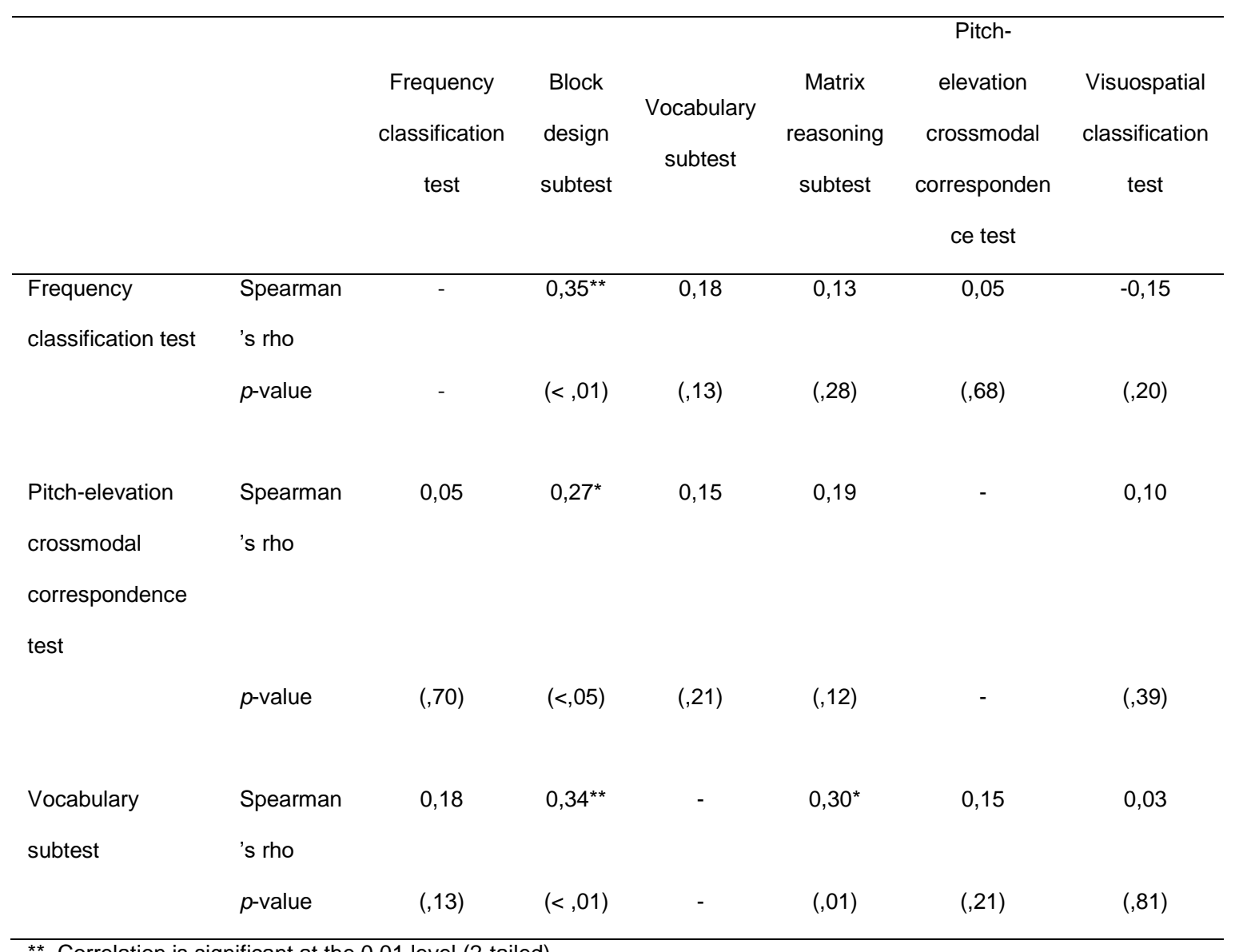

${ }^{\star *}$. Correlation is significant at the 0.01 level (2-tailed).

*. Correlation is significant at the 0.05 level (2-tailed). 
Audiospatial crossmodal associations in children

(a)

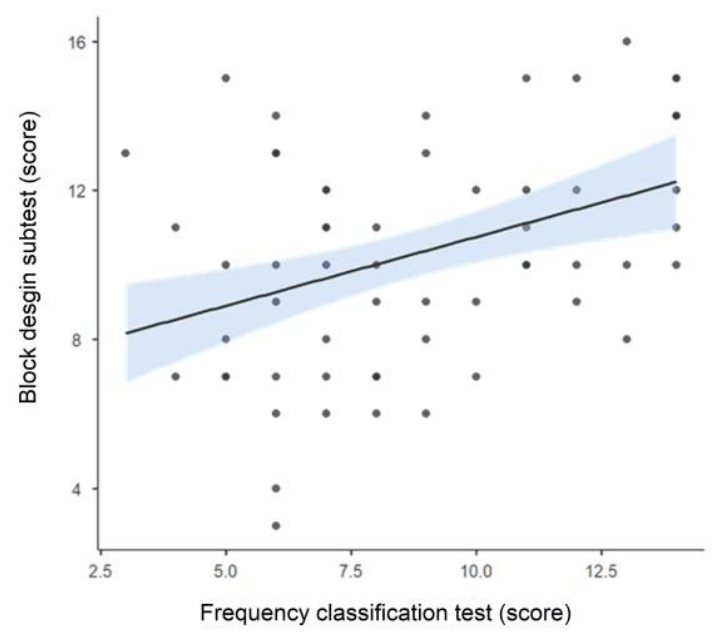

(b)

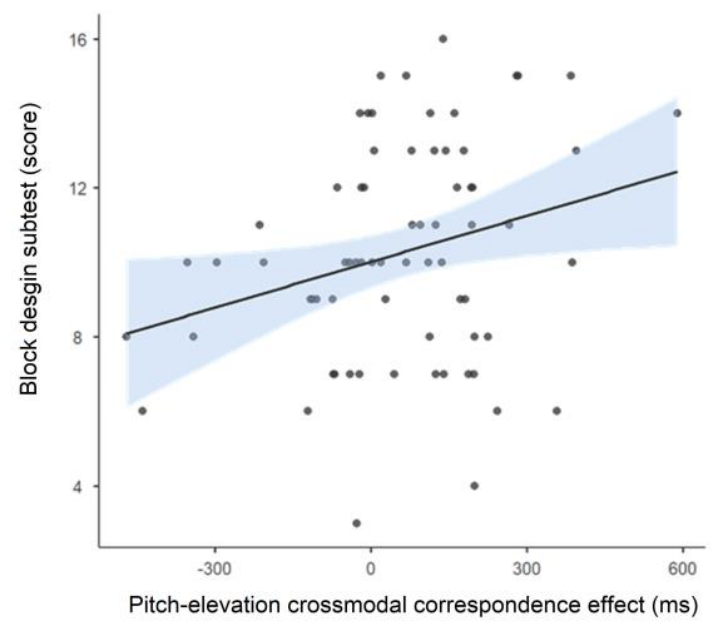

Figure 2. (a) Scatter plot showing the relationship between Frequency classification and Block design subtest punctuations. The blue shading indicates the standard error (b) Scatter plot for the relationship between Pitch-elevation crossmodal correspondence effect (Congruent minus incongruent reaction times in milliseconds) and Block design subtest scores. The blue shading indicates the standard error of the mean. 
Audiospatial crossmodal associations in children

\section{Discussion}

The present study examined the possible influence of the development and acquisition of linguistic and spatial abilities on the crossmodal correspondences between auditory pitch and spatial elevation. As predicted, our results indicated that children showed crossmodal correspondences between auditory and visuospatial features such as the one previously observed in adults (Rusconi et al. 2006). More specifically, children exhibited a crossmodal association between spatial elevation and pitch in the Pitchelevation crossmodal correspondence test. Their responses were faster and more accurate in the congruent trials than in the incongruent trials. Since this crossmodal effect has been previously observed in adults using similar methodologies (FernandezPrieto, Spence, Pons, \& Navarra, 2017; Rusconi et al., 2006), the current results support the idea that the crossmodal association between pitch and spatial elevation is already robustly present in childhood.

On the other hand, previous studies have demonstrated that children do not reach an optimal integration of information from different sensory systems until 8 to 12 years of age (Gori et al., 2008). Our results are in line with this previous evidence of fullydeveloped mechanisms of multisensory (audio-visual) integration at the age of 9 years (see also Barutchu et al., 2009; Gori et al., 2008).

The results of the present study also revealed a significant positive correlation between the performance in the Block design subtest and the Frequency classification test, and the Block design subtest and the Pitch-elevation crossmodal correspondence test. At a speculative level, this correlation may suggest that a more developed spatial cognitive system is associated with an enhancement of the previously reported space-related processing of pitch. In consonance with this idea, previous studies in adults suggest that deficits in pitch processing (e.g. amusia) are linked with cognitive mechanisms responsible for spatial representation processing (Douglas and Bilkey, 2007). Following 
the hypothesis of a common encoding of pitch and spatial elevation, the participants' results in the Visual-spatial classification and the Pitch-elevation crossmodal correspondence tests should also correlate. The reason for not observing such a pattern of results is possibly due to the fact that the Visual-spatial classification test was designed to ensure that participants were able to detect differences in spatial position between visual stimuli that were very obvious, which led to a high level (celling) performance (only $3.54 \%$ of errors in the congruent condition, and $9.33 \%$ in the incongruent condition) and possibly reduced the "space" for any reliable correlation between this and other measures.

Finally, it is important to emphasize that the tasks involving auditory processing did not correlate with either the Vocabulary or the Matrix reasoning subtests of the WISC-IV. Only the Block design subtest that measures spatial visualization and visuomotor skills, correlated positively with the task involving auditory pitch processing. However, although all children tested in the present study showed crossmodal correspondences, they did not demonstrate a correlational association between linguistic skills and pitchbased or audio-visual tasks. The absence of these correlations may suggest that the acquisition of linguistic skills does not play an essential role in the establishment of the links between auditory and visual-spatial features. These results may go in line with previous studies reporting the presence of crossmodal associations in prelinguistic infants (Dolscheid et al., 2014; Walker et al., 2010) and in speakers whose languages do not contain spatial-related labels to designate pitch (Parkinson et al., 2012). Interestingly enough, the previously reported influence of language on this crossmodal association may perhaps strengthen and modify these associations (Fernandez-Prieto et al., 2017; Martino \& Marks, 1999; Melara \& Marks, 1990). 
Audiospatial crossmodal associations in children

\section{Acknowledgements}

We would like to thank the students and teachers from Can Cantó Public School in Ibiza (Spain) that collaborated in the study. In particular, we would like to thank the school director Isabel Ferrer Arabí, for her help and collaboration. Also, we would like to thank Alexandria N. Holcomb for her helpful comments on an earlier version of the article. The present study was supported by a predoctoral fellowship from Institut de Recerca en Brain, Cognition \& Behaviour (IR3C; Universitat de Barcelona) \& Fundació Sant Joan de Déu to I.F.-P. and by grants PSI2012-39149 and PSI2010-20294 from Ministerio de Economía y Competitividad (Spanish Government) to J.N. and F.P., respectively. 
Audiospatial crossmodal associations in children

\section{References}

Barutchu, A., Crewther, D. P. and Crewther, S. G. (2009) 'The race that precedes coactivation: Development of multisensory facilitation in children', Developmental Science, 12(3), pp. 464-473. doi: 10.1111/j.1467-7687.2008.00782.x.

Deroy, O. et al. (no date) 'Unravelling the paradox of spatial pitch', in Hubbard, T. L. (ed.) Spatial biases in perception and cognition. Cambridge: Cambridge University Press.

Dolscheid, S. et al. (2014) 'Prelinguistic Infants Are Sensitive to Space-Pitch Associations Found Across Cultures', Psychological Science, (April), pp. 1-6. doi: $10.1177 / 0956797614528521$.

Douglas, K. M. and Bilkey, D. K. (2007) 'Amusia is associated with deficits in spatial processing', 10(7), pp. 915-921. doi: 10.1038/nn1925.

Fernandez-Prieto, I. et al. (2017) 'Does Language Influence the Vertical Representation of Auditory Pitch and Loudness?', i-Perception. SAGE Publications, 8(3), p. 2041669517716183 . doi: 10.1177/2041669517716183.

Fernández-Prieto, I., Navarra, J. and Pons, F. (2015) 'How big is this sound? Crossmodal association between pitch and size in infants', Infant Behavior and Development, 38, pp. 77-81. doi: 10.1016/j.infbeh.2014.12.008.

Foster, N. E. V. and Zatorre, R. J. (2010) 'Cortical structure predicts success in performing musical transformation judgments', Neurolmage, 53(1), pp. 26-36. doi: 10.1016/j.neuroimage.2010.06.042.

Foster, N. E. V and Zatorre, R. J. (2010) 'A role for the intraparietal sulcus in transforming musical pitch information', Cerebral Cortex, 20(6), pp. 1350-1359. doi: 10.1093/cercor/bhp199. 
Gallace, A. and Spence, C. (2006) 'Multisensory synesthetic interactions in the speeded classification of visual size.', Perception \& psychophysics, 68(7), pp. 11911203. doi: $10.3758 / \mathrm{BF} 03193720$.

Gori, M. et al. (2008) 'Young Children Do Not Integrate Visual and Haptic Form Information', Current Biology, 18(9), pp. 694-698. doi: 10.1016/j.cub.2008.04.036.

Lewkowicz, D. J. and Minar, N. J. (2014) 'Infants are not sensitive to synesthetic crossmodality correspondences: a comment on Walker et al. (2010).', Psychological science, 25(3), pp. 832-4. doi: 10.1177/0956797613516011.

Long, J. (1977) 'Contextual assimilation and its effect on the division of attention between nonverbal signals', Quarterly Journal of Experimental Psychology, 29(3), pp. 397-414. doi: 10.1080/14640747708400617.

Martino, G. and Marks, L. E. (1999) 'Perceptual and linguistic interactions in speeded classification : tests of the semantic coding hypothesis', 28(1978), pp. 903-924. doi: $10.1068 /$ p2866.

Melara, R. D. and Brien, T. P. O. (1987) 'Interaction Between Synesthetically Corresponding Dimensions', 116(4), pp. 323-336.

Melara, R. D. and Marks, L. E. (1990) 'Interaction among auditory dimensions: timbre, pitch, and loudness.', Perception \& psychophysics, 48(2), pp. 169-178. doi: 10.3758/BF03207084.

Mondloch, C. J. and Maurer, D. (2004) 'Do small white balls squeak? Pitch-object correspondences in young children', Cognitive, Affective, \& Behavioral Neuroscience, 4(2), pp. 133-136. doi: 10.3758/CABN.4.2.133.

Munar, E. et al. (2002) ‘El desarrollo de la audición humana', Psicothema, 14(2), pp. 247-254. 
Nardini, M., Bales, J. and Mareschal, D. (2016) 'Integration of audio-visual information for spatial decisions in children and adults', Developmental Science, 19(5), pp. 803816. doi: $10.1111 /$ desc. 12327.

Nava, E., Grassi, M. and Turati, C. (2016) 'Audio-visual, visuo-tactile and audio-tactile correspondences in preschoolers', Multisensory Research, 29(1-3), pp. 93-111. doi: 10.1163/22134808-00002493.

Occelli, V., Spence, C. and Zampini, M. (2009) 'Compatibility effects between sound frequency and tactile elevation.', Neuroreport, 20(8), pp. 793-7. doi:

10.1097/WNR.0b013e32832b8069.

Parkinson, C. et al. (2012) 'Associations between auditory pitch and visual elevation do not depend on language : Evidence from a remote population', 41, pp. 854-861. doi: $10.1068 /$ p7225.

Rusconi, E. et al. (2006) 'Spatial representation of pitch height : the SMARC effect', 99, pp. 113-129. doi: 10.1016/j.cognition.2005.01.004.

Sonnadara, R. R. et al. (2009) 'Spatial Properties of Perceived Pitch Influence on Reaching Movements', 507, pp. 503-507. doi: 10.1111/j.1749-6632.2009.04858.x. Spence, C. (2011) 'Crossmodal correspondences: A tutorial review’, (January), pp. 971-995. doi: 10.3758/s13414-010-0073-7.

Tillmann, B. et al. (2010) 'The Amusic Brain : Lost in Music, but Not in Space', 5(4), pp. 3-8. doi: 10.1371/journal.pone.0010173.

Walker, P. et al. (2010) 'Preverbal Infants' Sensitivity to Synaesthetic Cross-Modality Correspondences', Psychological Science, 21(1), pp. 21-25. doi: 10.1177/0956797609354734. 